\title{
Prescription pattern at outpatient department in a tertiary care hospital of Gujarat: a cross-sectional study
}

\author{
Nayana J. Gohil* \\ Department of Pharmacology, Shantabaa Medical College and General Hospital, Amreli, Gujarat, India
}

Received: 05 December 2019

Revised: 20 December 2019

Accepted: 21 December 2019

*Correspondence:

Dr. Nayana J Gohil,

Email: researchguide86@gmail.com

Copyright: (C) the author(s), publisher and licensee Medip Academy. This is an open-access article distributed under the terms of the Creative Commons Attribution Non-Commercial License, which permits unrestricted non-commercial use, distribution, and reproduction in any medium, provided the original work is properly cited.

\begin{abstract}
Background: Evaluation of drug prescription prototype is a significant feature of patient care, which also provides as an estimate of the quality of care provided. Thus, the current study was carried out to analyze the prescriptions of patients attending outpatient department (OPD) of a tertiary care teaching hospital.

Methods: Present cross-sectional study was performed in the OPD of our tertiary care teaching hospital. The study was carrying out for duration of two years. Data was obtained from 850 prescriptions. Data was analysed as per WHO prescribing indicators.

Results: Total 1700 prescriptions were analysed, that includes $55.8 \%$ males and $40.1 \%$ females. The highest numbers of prescriptions were from the age group of 18-40 (53.6\%) years of age. Average number of drugs in the current study was found to be (2.1). Percentage of come across with an antibiotic prescribed was $17.12 \%$. Percentage of come across with an injection prescribed was $11.81 \%$.

Conclusions: There is necessitate of recuperating the prescribing pattern by maintaining the number of medicines as little as possible, prescribing medicines by generic names, using medicines properly after selecting and deliberately keeping the charge of treatment low.
\end{abstract}

Keywords: Generic drugs, Medicine, Prescribing indicators, Rational prescribing

\section{INTRODUCTION}

The World Health Organization (WHO) has defined drug consumption research as the advertising, allocation, prescription and utilizes of drugs in a society, with particular importance on the resulting medical, social, and economic cost. WHO highlights two associated exertion concerning the drug position in the developing world: one out of three people living in the developing world are in require of necessary drugs though there are simultaneous superior rates of unsuitable 4 drug-use and drug resistance. ${ }^{1}$

Evaluation of drug prescription prototype is a significant feature of patient care, which also provides as an estimate of the quality of care provided. One recent study has resolute that prescribing reputation is dimensions need steady appraisal. The rational use of drugs is essential for an efficient and disciplined health-care system. However, irrational drug use, considered as a universal risk is mainly common in the developing countries due to irrational prescribing, stipulation, and management of medications. ${ }^{2,3}$

Medical prescription is a significant document of medico legal value too, that can be kept as proof in medico legal cases in court of law and thus should be cautiously and critically considered. More than $50 \%$ of all medicines global are prescribed, dispensed, or sold improperly and $50 \%$ of patients not succeed to take them perfectly. On 
the other hand, about one third of the world's population be deficient in access to critical medicines. ${ }^{4}$

Carry out sporadic studies of pattern of drug use in different hospital settings or patient populations is thus necessary to significantly examine the present hospital drug policies and to create suggestions based on different guidelines to get better upon the present drug usage pattern in the future, if required. ${ }^{5,6}$

The indicators of prescription in exercise calculate the recitals of health care provider in quite a few key magnitudes related to suitable use of drug. Therefore, the present study was carrying out to analyze the prescriptions of patients attending outpatient department (OPD) of a tertiary care teaching hospital.

\section{METHODS}

The study protocol was approved by the Institutional Ethics Committee. The research was conducted as a cross-sectional study in the OPD of Shantabaa Medical College \& General Hospital, Amreli, Gujarat. The study was conducted in Medical College and Research centre, for duration of two years from November 2017 to November 2019.

Inclusion criteria were the study included prescriptions collected randomly from all OPDs.

Well calibrated clinical pharmacists were involved in data collection on prescribing indicators. Every prescription was considered as single-patient come upon for calculation of the necessary parameters. Prescriptions of patients attending medical OPD and treated on outpatient basis for their disease were included irrespective of the co morbidities. Collection of data was done on the demographic particulars of age, gender, diagnosis, and the treatment prescribed which were bring up in the prescription. The prescribing indicators were studied in accordance to the standard guideline advocated by the WHO. Average number of drugs per meet: Average was considered by separating the total number of different drug products prescribed, by the number of encounters surveyed. Whether the patient really go the drugs was not considered applicable in calculating this indicator, percentage of encounters with an antibiotic prescribed. Percentage was calculated by dividing the number of patient encounters during which an antibiotic was prescribed, by the total number of encounters surveyed and expressed as a percentage. Percentage of drugs prescribed by generic name, percentage was calculated by dividing the number of drugs prescribed by generic name, by the total number of drugs prescribed and expressed as a percentage. Percentage of drugs prescribed from essential drugs list or formulary, percentage was calculated by dividing the number of products prescribed which were on the essential drugs list or local formulary, by the total number of products prescribed and multiplied by 100 . Percentage of encounters with an injection prescribed, percentage was calculated by dividing the number of patient encounters during which an injection was prescribed, by the total number of encounters surveyed, multiplied by 100 .

For analysis of average number of drugs per encounter, combinations were considered as single drug and for the rest of indicators combination were split into individual drugs and counted separately. Multivitamin prescription was counted as one, for example B-complex.

\section{Statistical analysis}

The recorded data was compiled and entered in a spreadsheet computer program (Microsoft Excel 2007) and then exported to data editor page of SPSS version 15 (SPSS Inc., Chicago, Illinois, USA). Descriptive statistics included computation of percentages.

\section{RESULTS}

The male to female ratio was found to be $1.5: 1$. Total of 1700 prescriptions were included in the study. Total of 980 prescriptions were of male and 720 prescriptions were of females. The male proportions were slightly more than females. Majority of the patients belongs to the age group of 40-60 years (Table 1 and 2).

Table 1: Distribution of gender.

\begin{tabular}{|ll|}
\hline Gender & No. of prescription \\
\hline Male & 980 \\
\hline Female & 720 \\
\hline
\end{tabular}

Table 2: Distribution of age group.

\begin{tabular}{|ll|}
\hline Age group in years & No of prescription \\
\hline$<\mathbf{1 8}$ & 104 \\
\hline $\mathbf{1 9 - 3 9}$ & 906 \\
\hline $\mathbf{4 0 - 5 9}$ & 594 \\
\hline $\mathbf{6 0 - 7 9}$ & 86 \\
\hline
\end{tabular}

It was found that a total number of 3899 drug products had been prescribed in the 1700 patient encounters, and thus, the average number of drugs per prescription was 2.10 and the standard deviation was 1.45 . Moreover, the median number of drugs per prescription was 3 , which unlike the mean, would not be unduly influenced by the outliers. Overall, the study revealed a higher value for this indicator than the reference standard. Analyzing the patient encounters lacking the data for specified variables, it was found, $(88.32 \%)$ prescriptions did not have any generic names, whereas only 17 patient encounters lacked at least one drug from essential drugs list. It was evident that $82.17 \%$ prescriptions did not have any antibiotics and likewise, a high percentage of $89.45 \%$ did not have any injections prescribed. 
It was interesting to find that among the total drug products prescribed, the highest percentage of prescribed drugs were supplements which ranked first followed by the antidiabetic, antiplatelet, hypolipidemic, and antihypertensive drugs, whereas the overall percentage of antibiotics prescribed was only $17.56 \%$, and hence lower in comparison to the former groups of drugs.

Table 3: Trend in prescribing.

\begin{tabular}{|ll|}
\hline Indicator & Result \\
\hline Average number of drugs per encounter & 2.10 \\
\hline $\begin{array}{l}\text { Percentage of drugs prescribed by generic } \\
\text { name }\end{array}$ & 11.68 \\
\hline $\begin{array}{l}\text { Percentage of encounters with an } \\
\text { antibiotic prescribed }\end{array}$ & 17.46 \\
\hline $\begin{array}{l}\text { Percentage of encounters with an injection } \\
\text { prescribed }\end{array}$ & 11.56 \\
\hline
\end{tabular}

\section{DISCUSSION}

Standard number of drugs per encounter in the present study was found to be 2.1. Another study conducted by Geetah et al in tertiary care hospital, Chennai reported 3.68 which are higher from our observation. ${ }^{4}$ Slightly lower results had been observed from a study conducted in a rural hospital in Jalna i.e. 1.82.

The high average number of drug products per prescription more than the WHO reference range is affectionate of a high degree of polypharmacy prevalent in our study locale. The reasons that could be ascribed to this is the change in the epidemiological trend of violently increased prevalence of non-communicable diseases such as diabetes, hypertension, dyslipidemia, and coronary artery disease which are often synchronized contributing to the require for treating numerous disease entities in the same patient concurrently. ${ }^{7}$ Concomitance of such cardio metabolic circumstances in a solitary patient further entails the mandatory for prescribing more than a single drug for a known clinical indication which is frequently opposed to treatment in the face of several diseases presenting simultaneously. Present study also stands witness to this fact as a high percentage of participants $(68.6 \%)$ had the analysis of non-communicable disease with diabetes ranking highest with $34.4 \%$.

The percentage of drugs prescribed by generic name in current study was $11.68 \%$ which is too little contrast with the standard derived to serve as perfect, which is $100 \%$. Wang et al described that elevated the doctor's teaching and training experience, the proportion of drugs that they prescribed by generic names showed a decline. ${ }^{8}$ furthermore, attitudinal difference have also been shown to be among consultant in low- and middle-income countries contrast to those in high-income countries. ${ }^{9}$ The probable description for the low percentage of generic prescribing could be owing to frequent and influential encouragement of the propriety products by pharmaceutical companies and in certain case, clinicians are bound to concede to the resolve of wealthy patients demanding pacesetter drugs for treatment. ${ }^{3}$

In the present study $11.93 \%$ of encounters had an injection prescribed which is somewhat higher than Bhatnagar et al (10\%) but lower than the study carry out (14\%) and another study conducted by Simpson et al reported $38 \%$ and Mittal et al $80 \%$ use of injectables. ${ }^{10-12}$ Injectable drugs are connected with trouble of administration and medication errors. Therefore, the require to switch over to other routes of administration as soon as possible needs to be emphasised.

\section{CONCLUSION}

Our study on prescribing indicators has clearly delineated that the prescribing practices for antibiotics and injections are appropriate and rational; conformity to essential drugs list is considerably good, though scope for improvising is certainly evident. Appropriate measures to reduce polypharmacy and increase generic prescribing by clinicians have to be implemented by the administrative team and policymakers to ensure rational and safe prescribing.

\section{Funding: No funding sources}

Conflict of interest: None declared

Ethical approval: The study was approved by the Institutional Ethics Committee

\section{REFERENCES}

1. Page RL, II SAL, Bryant LL, Ruscin JM. Inappropriate prescribing in the hospitalized elderly patient: defining the problem, evaluation tools, and possible solutions. Clinical Interventions Aging. 2010;5:75-87.

2. Abubakar AR, Chedi BA, Simbak NB, Haque M: Medication error: The role of health care professionals, sources of error and prevention strategies. J Chem Pharm Res. 2014;6:646-51.

3. Shanmugapriya S, Saravanan T, Rajee SS, Venkatrajan R, Thomas PM. Drug prescription pattern of outpatients in a tertiary care teaching hospital in Tamil Nadu. Perspect Clin Res. 2018;9:133-8.

4. Geetha P, Vijayalakshmi P, Tilak S, Maheswaran A, Narayanan N. Prescription analysis to evaluate the rational use of drugs by using who health care indicators. Int J Multidiscip Res Dev. 2015;2:358-60.

5. Kaur S, Rajagopalan S, Kaur N, Shafiq N, Bhalla A, Pandhi P, Malhotra S. Drug utilization study in medical emergency unit of a tertiary care hospital in North India. Emerg Med Int. 2014;2014:973578.

6. Lohr KN, Eleazer K, Mauskopf J. Health policy issues and applications for evidence-based medicine and clinical practice guidelines. Health Policy. 1998;46:1-19. 
7. Gowthami B, Spurthi T. Drug utilization evaluation of antibiotics in general medicine department of a tertiary care hospital. Value Health. 2016;19:824.

8. Wang $\mathrm{H}, \mathrm{Li} \mathrm{N}$, Zhu $\mathrm{H}, \mathrm{Xu} \mathrm{S}, \mathrm{Lu} \mathrm{H}$, Feng Z. Prescription pattern and its influencing factors in Chinese county hospitals: a retrospective crosssectional study. PloS one. 2013;8:e63225.

9. Hassali MA, Wong ZY, Alrasheedy AA, Saleem F, Yahaya AHM, Aljadhey H. Perspectives of physicians practicing in low and middle income countries towards generic medicines: a narrative review. Health Policy. 2014;117:297-310.

10. Bhatnagar T, Mishra C, Mishra R: Drug prescription practices: a household study in rural Varanasi. Indian J Prev Soc Med. 2003;34:33-9.
11. Simpson G, Naveen Chaudhary G. Comparative analysis of prescription writing by teaching and nonteaching clinicians in and around Guntur. Int $\mathbf{J}$ Recent Trends Sci Tech. 2012;5:100-3.

12. Mittal N, Mittal R, Singh I, Shafiq N, Malhotra S. Drug utilisation study in a tertiary care center: Recommendations for improving hospital drug dispensing policies. Indian J Pharm Sci. 2014;76:308.

Cite this article as: Gohil NJ. Prescription pattern at outpatient department in a tertiary care hospital of Gujarat: a cross-sectional study. Int J Basic Clin Pharmacol 2020;9:53-6. 